\title{
Incidence and Clinical Characteristics of Lower Urinary Tract Symptoms as a Presenting Symptom for Patients with Newly Diagnosed Bladder Cancer
}

Ryan W. Dobbs, Lee A. Hugar, Louis M. Revenig, Usama Al-Qassab, John A. Petros, Chad W. Ritenour, Muta M. Issa, Daniel J. Canter

Department of Urology, Atlanta Veterans' Affairs Medical Center and Emory University School of Medicine, Atlanta, Georgia, USA

\section{ABSTRACT}

Purpose: The incidence of lower urinary tract symptoms (LUTS) as the sole presenting symptom for bladder cancer has traditionally been reported to be low. The objective of this study was to evaluate the prevalence and clinical characteristics of newly diagnosed bladder cancer patients who presented with LUTS in the absence of gross or microscopic hematuria.

Materials and Methods: We queried our database of bladder cancer patients at the Atlanta Veteran's Affairs Medical Center (AVAMC) to identify patients who presented solely with LUTS and were subsequently diagnosed with bladder cancer. Demographic, clinical, and pathologic variables were examined.

Results: 4.1\% (14/340) of bladder cancer patients in our series presented solely with LUTS. Mean age and Charlson Co-morbidity Index of these patients was 66.4 years (range $=52-83$ ) and 3 (range $=0-7$ ), respectively. Of the 14 patients in our cohort presenting with LUTS, 9 (64.3\%), 4 (28.6\%), and 1 (7.1\%) patients presented with clinical stage Ta, carcinoma in Situ (CIS), and T2 disease. At a median follow-up of 3.79 years, recurrence occurred in 7 (50.0\%) patients with progression occurring in $1(7.1 \%)$ patient. $11(78.6 \%)$ patients were alive and currently disease free, and 3 (21.4\%) patients had died, with only one (7.1\%) death attributable to bladder cancer.

Conclusions: Our database shows a $4.1 \%$ incidence of LUTS as the sole presenting symptom in patients with newly diagnosed bladder cancer. This study suggests that urologists should have a low threshold for evaluating patients with unexplained LUTS for underlying bladder cancer.

\section{ARTICLE INFO}

Key words:

Ureteral Obstruction; Urinary

Bladder Neoplasms; Lower

Urinary Tract Symptoms; Early

Detection of Cancer

Int Braz J Urol. 2014; 40: 198-203

Submitted for publication:

July 22, 2013

Accepted after revision:

March 05, 2014

\section{INTRODUCTION}

In 2012, there will be an estimated 73,510 new diagnoses of bladder cancer with approximately 15,000 deaths attributable to this cancer in the United States (1). Bladder cancer is strongly associated with exposure to smoking, environmental toxins, and aging and peaks in prevalence and incidence in the 8th decade of life (2). Typically, the majority of patients with bladder cancer present with gross painless hematuria, with estimates of this presentation accounting for up to 85\% of patients at initial diagnosis $(3,4)$. Asymptomatic microhematuria is the next most common presenting symptom for bladder 
cancer with up to $10 \%$ of patients evaluated for the presence of microscopic hematuria being diagnosed with bladder cancer (5). The remaining small subset of newly diagnosed bladder cancer patients will present with symptoms that mimic other common urological conditions, such as urinary tract infections and/or lower urinary tract symptoms (LUTS).

Refractory LUTS are a well-recognized initial presenting symptom in a small percentage of patients with newly diagnosed bladder cancer. Traditionally, patients who present with refractory LUTS are thought to have carcinoma in-situ (CIS) at diagnosis (6). Previous studies have shown that up to one fourth of patients with CIS will present with irritative voiding symptoms including frequency, urgency and dysuria (7).

In this study, we sought to examine the incidence of LUTS without hematuria as an initial presenting symptom for bladder cancer in a high risk population, namely an elderly (2), veteran (8), male (2) cohort with significant tobacco (9) and chemical/environmental (10) exposures. Further, we sought to compare the clinical and pathologic data between patients who presented with LUTS compared to those who presented with gross or microscopic hematuria.

\section{MATERIALS AND METHODS}

This study was approved by the institutional review board of the Atlanta Veteran's Affairs Medical Center (AVAMC). Patients with bladder cancer at the AVAMC were identified through a comprehensive review of urologic surgical records, pathology records, International Classification of Diseases (ICD)-9 coding, and the AVAMC cancer registry from 2000-2012. Pertinent clinical and demographic data were retrospectively collected from patient records, including presenting symptomatology that prompted initial clinical workup. In the database, presenting symptomatology was divided into four categories based on the clinical records in patients' charts: gross hematuria, microscopic hematuria, lower urinary tract symptoms (LUTS) and incidental detection. Other demographic and clinical variables collected included patient age, race, associated co-morbidities, tobacco use, relevant pre and post-operative laboratory values, histologic type, stage and grade based on transurethral resection/ bladder biopsy of bladder lesion.

Patients in our bladder cancer database were classified as presenting with lower urinary tract symptoms based on their seeking/being referred for a urologic consultation for irritative and/ or obstructive voiding symptoms in the absence of microscopic or gross hematuria. As a part of their initial and ongoing evaluation, these patients were further evaluated with the International Prostate Symptom Score (IPSS) questionnaire to assess the severity of their symptoms. Patient IPSS scores were calculated based on a series of questions assessing their urinary symptoms, scored from 0 ("not bothersome") to 4 ("very bothersome") for each question. The patient quality of life score was obtained from the final IPSS quality of life question regarding the patient's overall satisfaction with their urinary symptoms, ranging from 0 "delighted" to 6 "terrible". No specific numerical IPSS cut-off was utilized for inclusion of patients in the LUTS group - it was simply the presenting symptom for this subset of patients who were ultimately diagnosed with bladder cancer. Patients included in this analysis were all identified as a newly diagnosed with bladder cancer between 2000 and 2012. Patients with a previous history of bladder cancer or a diagnosis of bladder cancer from an outside facility were excluded from this analysis. Patients were classified as presenting with LUTS as long as they did not have a history of either gross or microscopic hematuria $(\geq 3 \mathrm{RBC}$ per HPF) or a history of bladder carcinoma. Categorical variables were analyzed using a chi-square or Fisher's exact test when appropriate. Continuous variables were analyzed using either the Student's t-test for comparing of means or the Wilcoxon rank sums test for comparing medians. All statistical tests were two-sided with the significance level $\alpha$ set to 0.05. Statistics were performed using SAS 9.3 (SAS Institute, Cary, NC).

\section{RESULTS}

A total of 340 patients were identified as presenting with a new diagnosis of bladder cancer to the AVAMC between 2000 and 2012. These patients were comprised of 337 male (99.1\%) and 3 female $(0.9 \%)$ patients with a mean/median age at diagnosis 
of 67.7 and 67 years (range $=30-93$ ), respectively. The patient cohort consisted of 74 (21.8\%) African-American patients and 263 (77.4\%) non-African-American patients. 266 (78.2\%), 40 (11.8\%), 20 (5.9\%), and 14 (4.1\%) patients presented with gross hematuria, microhematuria, incidentally, and LUTS. The clinical and demographic information for the 14 patients presenting with LUTS are shown in Table-1 and compared to the remaining cohort of 326 patients. Both groups did not significantly vary in their composition by gender $(p=1.00)$, age $(p$
$=0.63)$, BMI $(p=0.65)$, pack-years of smoking $(p$ $=0.79$ ) or Charlson Co Morbidity Index scores ( $p$ $=0.76$ ). Patients presenting with LUTS did present with statistically significant worse IPSS $(p<0.001)$, Bother $(\mathrm{p}<0.001)$, and QOL ( $\mathrm{p}=0.049)$ scores as compared to the other groups as shown in Table-1.

Mean and median follow-up for the entire cohort was 3.1 and 2.3 years respectively. Pathological characteristics of the two groups are presented in Table-2, patients presenting with LUTS were significantly more likely to present with CIS

Table 1 - Demographic and clinical presentation for LUTS and Non-LUTS patient groups.

\begin{tabular}{lccc}
\hline Variable & LUTS $(\mathrm{n}=14)$ & Non-LUTS $(\mathrm{n}=326)$ & P-value \\
\hline Gender (Men) & $14(100 \%)$ & $323(99.1 \%)$ & $1.00 \dagger$ \\
Mean Age \pm SD, Range & $66.4 \pm 9.3,52-83$ & $67.8 \pm 10.3,30-93$ & $0.63 \ddagger$ \\
Median BMI \pm IQR, Range & $28.2 \pm 3.1,21-51$ & $30.0 \pm 6.8,17-51$ & $0.65 \S$ \\
Median Pack Years \pm IQR, Range & $45.0 \pm 57.0,0-70$ & $40.0 \pm 44.0,0-210$ & $0.79 \S$ \\
Median CCI \pm IQR, Range & $3.5 \pm 5.0,0-7$ & $3.0 \pm 5.0,0-16$ & $0.76 \S$ \\
Mean IPSS \pm SD & $24.0 \pm 4.6$ & $13.0 \pm 9.4$ & $<0.001 \ddagger$ \\
Mean Bother \pm SD & $20.4 \pm 3.8$ & $10.7 \pm 8.2$ & $<0.001 \ddagger$ \\
Mean QOL \pm SD & $4.2 \pm 1.7$ & $3.0 \pm 1.8$ & $0.049 \ddagger$ \\
\hline
\end{tabular}

† = Fisher's exact test; $\mathbf{\ddagger}$ = Student's t-test; $\S$ = Wilcoxon rank sums test; $\mathbf{S D}=$ standard deviation; $\mathbf{I Q R}$ = Interquartile range

Table 2 - Pathologic Characteristics for LUTS and Non-LUTS patient groups.

\begin{tabular}{lccc}
\hline & LUTS $(\mathrm{n}=14)$ & Non-LUTS $(\mathrm{n}=326)$ & P-value \\
\hline CIS (only) & $3(21.4 \%)$ & $12(3.7 \%)$ & $0.02 \dagger$ \\
Ta & $10(71.4 \%)$ & $184(56.4 \%)$ & $0.27 \ddagger$ \\
T1 & $0(0.0 \%)$ & $69(21.2 \%)$ & $0.08 \dagger$ \\
Muscle Invasive (Stage $\geq$ T2) & $1(7.1 \%)$ & $57(17.7 \%)$ & $0.48 \dagger$ \\
Grade (high) & $7(50.0 \%)$ & $197(60.4 \%)$ & $0.44 \ddagger$ \\
Recurrence & $7(50.0 \%)$ & $131(40.2 \%)$ & $0.46 \ddagger$ \\
Mean No. Recurrence & 0.57 & 0.68 & $0.56 \S$ \\
Mean No. Recurrences (among those with a recurrence) & 1.1 & 1.7 & $0.009 \S$ \\
No. Progression & $1(7.1 \%)$ & $25(7.7 \%)$ & $1.00 \dagger$ \\
\hline
\end{tabular}

† = Fisher's exact test; $¥$ = chi square test; $\S=$ Student's t-test 
in the LUTS group compared to the non-LUTS group (21.4\% versus 3.7\%, $\mathrm{p}=0.02)$. Conversely, patients who presented with non-LUTS were more likely to experience a disease recurrence than patients who presented with LUTS (1.7 mean recurrences versus $1.1, \mathrm{p}=0.009)$. At last follow-up, $11(78.6 \%)$ of patients presenting with LUTS were alive without disease and $3(21.4 \%)$ patients had died. Of the patients who died, one death (7.1\%) was attributed to bladder cancer. This patient initially presented with CIS and declined further treatment despite multiple positive cytologies and evidence of progression of disease. Compared to patients that did not present with lower urinary tract symptoms, there were no significant differences in progression and bladder-cancer related death rates.

\section{DISCUSSION}

In our study, patients who presented solely with LUTS comprised 4.1\% (14/340) of newly diagnosed bladder cancer patients. Within this group of patients, there was a statistically significant $(p=0.02)$ greater incidence of clinical CIS upon presentation as compared to the non-LUTS patients. While there was a significant portion of LUTS patients presenting with CIS, the majority of patients who presented with LUTS 71.4\% (10/14) did not have CIS. These results indicate that while clinicians should have a strong clinical suspicion for potential CIS lesions, the majority of patients with LUTS who present with bladder cancer will have a papillary lesion.

Lower urinary tract symptoms (LUTS) comprise a constellation of storage, voiding and post-micturition symptoms that are common in both men and women and rise in prevalence with age (11). In men, LUTS are typically attributed to the presence of benign prostatic hyperplasia (BPH) although LUTS may occur independently of $\mathrm{BPH}$ (12). Like bladder cancer, LUTS and BPH are strongly associated with aging; large population studies estimate the overall prevalence of LUTS as $62.5 \%$ of men over the age of 40 and $80.7 \%$ of men over the age of 60 (13).

Estimates for the incidence of LUTS as a presenting symptom for bladder cancer varies widely due to heterogeneous patient populations and varied definitions for LUTS/voiding dysfunction. In a small patient cohort of 92 patients, voiding problems in conjunction with microhematuria was a presenting symptom in 18\% of newly diagnosed bladder cancer patients (14). Similarly, in a larger study of 1,000 patients, frequency and dysuria $(6.0 \%)$, difficult or poor stream (3.5\%) and acute retention (4.0\%) were presenting symptoms for bladder cancer albeit with significant co-existing (35-41\%) hematuria in each of these groups (15). In one study, 4 of 22 CIS patients were characterized as presenting with LUTS without hematuria (16). Also, for 481 newly presenting patients, $97.0 \%$ of patients presented with some form of hematuria with frequency and dysuria comprising the next most common presentation (17). In another study, "cystitis" was implicated as the primary symptom for patients with newly diagnosed bladder cancer in 12\% of patients $(12 / 100)$ and "obstructive symptoms" were implicated for 3\% of patients (3/100) (18). While these studies note that many bladder cancer patients present with irritative or obstructive symptoms, they do not evaluate the oncological outcomes of patients who present solely with LUTS. Furthermore, the irritative and obstructive presentations are often contaminated with either microscopic or gross hematuria. Our study provides new data by estimating the incidence of bladder cancer patients who presented with isolated LUTS as $4.1 \%$ as well as specifically reporting these patients' oncological outcomes.

The United States Preventative Services Task Force (USPSTF) current recommendations are that there is insufficient evidence to assess the harms and benefits of screening for bladder cancer in asymptomatic patients (19). Similarly, no major organization including the American Association of Family Physicians (AAFP), European Association of Urology (EAU) or The American Cancer Society (ACS) currently recommends screening asymptomatic patients for bladder cancer (19). Our patient cohort represents a unique patient population where screening is clinically indicated; namely, this patient population has a number of risk factors that have been associated with an increased risk of developing bladder cancer: tobacco use, chemical exposures, etc. As such, it appears reasonable for urologists to have a low threshold 
for performing cystoscopy for LUTS, especially in a high-risk patient population.

A potential concern from our study is that the use of non-invasive urine cytology may be preferential to flexible cystoscopy for the surveillance and identification of potential bladder cancer patients presenting with LUTS. Previous studies have indicated that the yield of urine cytology in the workup for patients with LUTS without hematuria has been low (20). One of the major problems with urine cytology is that this test lacks sensitivity for low and intermediate grade malignancies that comprise the majority of bladder cancer patients as well as the majority of patients in our study.

Another potential weakness of our study was an inability to evaluate for the number of cystoscopies that may have been performed to identify our patient cohort. The value of cystoscopy for evaluation of LUTS symptoms has also been evaluated in a number of previous studies. Goldberg et al. evaluated 1,584 women undergoing cystoscopy for LUTS and noted that 10 patients $(0.63 \%)$ were identified with bladder cancer (21). Of note, $60 \%(6 / 10)$ of the bladder cancer patients initially had a normal dipstick evaluation for hematuria (21). In a study by Weiss et al. evaluating patients for refractory overactive bladder without hematuria, 8 patients were identified with bladder cancer from a total of 1,420 patients undergoing cystoscopy for a diagnostic yield of 0.6\% (22). Despite all these concerns, this study does provide some insight into the incidence and clinical course of newly diagnosed bladder cancer patients who present solely with LUTS.

\section{CONCLUSIONS}

In our patient cohort, a small percentage of patients with newly diagnosed bladder cancer present with LUTS without gross or microscopic hematuria. Despite a higher incidence of CIS compared to patients with other presenting symptoms, the majority of patients with LUTS presented with Ta lesions. In high risk populations with significant tobacco exposure and other risk factors, clinicians should have a low threshold for performing an endoscopic evaluation of patients' lower urinary tracts when significant, difficult to treat LUTS exist.

\section{ABBREVIATIONS}

AVAMC = Atlanta Veteran Affairs Medical Center AUA $=$ American Urological Association

$\mathrm{RBC}=$ Red Blood Cell

LUTS = Lower Urinary Tract Symptoms

$\mathrm{AA}=$ African American

IQR = Interquartile Range

$\mathrm{SD}=$ Standard Deviation

\section{CONFLICT OF INTEREST}

None declared.

\section{REFERENCES}

1. Siegel R, Naishadham D, Jemal A: Cancer statistics, 2012. CA Cancer J Clin. 2012; 62: 10-29.

2. Parkin DM: The global burden of urinary bladder cancer. Scand J Urol Nephrol Suppl. 2008; 218: 12-20.

3. Khadra MH, Pickard RS, Charlton M, Powell PH, Neal DE: A prospective analysis of 1,930 patients with hematuria to evaluate current diagnostic practice. J Urol. 2000; 163: 524-7.

4. Alishahi S, Byrne D, Goodman CM, Baxby K: Haematuria investigation based on a standard protocol: emphasis on the diagnosis of urologicalmalignancy. J R Coll Surg Edinb. 2002; 47: 422-7.

5. Sultana SR, Goodman CM, Byrne DJ, Baxby K: Microscopic haematuria: urological investigation using a standard protocol. Br J Urol. 1996; 78: 691-6; discussion 697-8.

6. Kaufman DS, Shipley WU, Feldman AS: Bladder cancer. Lancet. 2009; 374: 239-49.

7. Farrow GM, Utz DC, Rife CC, Greene LF: Clinical observations on sixty-nine cases of in situ carcinoma of the urinary bladder. Cancer Res. 1977; 37: 2794-8.

8. Reulen RC, Kellen E, Buntinx F, Brinkman M, Zeegers MP: A meta-analysis on the association between bladder cancer and occupation. Scand J Urol Nephrol Suppl. 2008; 218: 6478.

9. Freedman ND, Silverman DT, Hollenbeck AR, Schatzkin A, Abnet CC: Association between smoking and risk of bladder cancer among men and women. JAMA. 2011; 306: 737-45. Erratum in: JAMA. 2011; 306: 2220.

10. Letašiová $S$, Medve'ová A, Šovčíková A, Dušinská M, Volkovová K, Mosoiu C, et al.: Bladder cancer, a review of the environmental risk factors. Environ Health. 2012; 11: S11. 
11. Boyle P, Robertson C, Mazzetta C, Keech M, Hobbs FD, Fourcade $\mathrm{R}$, et al.: The prevalence of lower urinary tract symptoms in men and women in four centres. The UrEpik study. BJU Int. 2003; 92: 409-14.

12. Kuo HC: Pathophysiology of lower urinary tract symptoms in aged men without bladder outlet obstruction. Urol Int. 2000; 64: 86-92.

13. Irwin DE, Milsom I, Hunskaar S, Reilly K, Kopp Z, Herschorn $S$, et al:: Population-based survey of urinary incontinence, overactive bladder, and other lower urinary tractsymptoms in five countries: results of the EPIC study. Eur Urol. 2006; 50: 1306-14; discussion 1314-5.

14. Boman $\mathrm{H}$, Hedelin $\mathrm{H}$, Jacobsson $\mathrm{S}$, Holmäng S: Newly diagnosed bladder cancer: the relationship of initial symptoms, degree of microhematuria and tumor marker status. J Urol. 2002; 168: 1955-9.

15. Gardner BP, Doyle PT: Symptoms of bladder carcinoma. J R Coll Gen Pract. 1987; 37: 367.

16. Ek A, Hellsten S, Henrikson H, Idwall I, Lindholm CE, Lindholm $\mathrm{K}$, et al.: Intravesical adriamycin therapy in carcinoma in situ of the urinary bladder. Scand J Urol Nephrol. 1984; 18: 131-4.
17. Gupta $P$, Jain $M$, Kapoor R, Muruganandham K, Srivastava $A$, Mandhani $A$ : Impact of age and gender on the clinicopathological characteristics of bladder cancer. Indian J Urol. 2009; 25: 207-10.

18. Stower MJ: Delays in diagnosing and treating bladder cancer. Br Med J (Clin Res Ed). 1988; 296: 1228-9.

19. Moyer VA; U.S. Preventive Services Task Force: Screening for bladder cancer: U.S. Preventive Services Task Force recommendation statement. Ann Intern Med. 2011; 155: 246-51. Erratum in: Ann Intern Med. 2011; 155: 408.

20. Potter JM, Quigley M, Pengelly AW, Fawcett DP, Malone PR: The role of urine cytology in the assessment of lower urinary tract symptoms. BJU Int. 1999; 84: 30-1.

21. Goldberg RP, Sherman W, Sand PK: Cystoscopy for lower urinary tract symptoms in urogynecologic practice: the likelihood of finding bladder cancer. Int Urogynecol J Pelvic Floor Dysfunct. 2008; 19: 991-4.

22. Weiss J, Blaivas J, Somaroo C, Panagopoulos G, Rutman M. Refractory Overactive Bladder Without Hematuria: A Presenting Symptom of Bladder Cancer. ICS/IUGA Annual Meeting. Toronto, 2010.

\section{Correspondence address:} Daniel Canter, MD Department of Urology Einstein Healthcare Network Urologic Institute of Southeastern Pennsylvania 60 Township Line Road Elkins Park, PA 19027, USA

Fax: + 1215 663-7089 E-mail: danieljcanter@gmail.com 